\title{
DEBATIENDO CON NORBERT ELIAS: ENTRE EL YO Y EL NOSOTROS
}

\section{Fermín Bouza}

Universidad Complutense de Madrid

\section{LA TENSION METODOLOGICA}

En la obra de Norbert Elias hay dos preocupaciones básicas que pueden convertirse en obsesiones cuando son objeto central de su texto: el evolucionismo como forma natural de la sociología (una «sociología de los procesos», como él dice) y el dilema metodológico individualismo/holismo, que toma diversas formas, como la que aquí me convoca, que atañe a los «cambios en el equilibrio entre el yo y el nosotros». Decir que son dos obsesiones es lo mismo que decir que, para Elias, hay ahí en esos temas diversos agujeros negros por los que la sociología contemporánea pierde buena parte de sus energías analíticas: de un lado, el estructuralismo acrónico de raíz durkheimiana y, de otro, la díada que forman el holismo sin sujeto (sin individuo) y el individualismo sin sociedad o sin grupo. Son cuestiones históricas y recurrentes en el debate metodológico, y resumen buena parte de los temas que los científicos sociales se han planteado en los niveles más abstractos de su tarea. La diferencia entre Elias y la reflexión metodológica convencional es que en ésta los problemas aparecen flotando en el cielo de la irrelevancia gaseosa, ejercicios de retórica al margen de la vida científica real, mientras que en Elias son parte de su difícil avance científico hacia la tierra prometida del neoevolucionismo que representa su sociología de los procesos, obstáculos metodológicos que intenta superar resituando el problema en su historia. 
La obra de Elias se percibe así sobre un fondo que la destaca, construido ese fondo con esas obsesiones metodológicas recurrentes que con frecuencia se convierten en objeto principal de sus textos, relegando a la cuestión concreta que les dio origen. Esas obsesiones no pueden ser ajenas al lector, que se encuentra con ese apasionado infierno particular del sociólogo, en cuyas llamas acaba quemándose, irremisiblemente, porque no es fácil coincidir a veces con sus planteamientos y porque, de todas formas, sus planteamientos son tan sugestivos que nos van ganando a pesar de nuestras resistencias. Elias pertenece al género de científicos sociales que dice cosas, más allá de la irrelevancia de una parte de la teoría social contemporánea de diverso origen ideológico, y las dice con fundamento.

Su espina metodológica más aguda es la que deriva de la constatación de que el individuo es el punto de partida empírico de toda sociología bien fundada y, sin embargo, él se sitúa en un historicismo cuya inercia le podría llevar a ignorar tal arranque empírico: demostrar que él no prescinde ni del individuo ni de la sociedad, y que está a igual distancia del holismo y del individualismo para acatar las dos realidades (individuo y sociedad) que definen el trabajo sociológico, es su objetivo explícito o implícito, y es el tema central de su trabajo, aparezca o no enunciado de esa forma.

En el artículo en el que ahora me voy a centrar, "Cambios en el equilibrio entre el yo y el nosotros» (N. Elias, 1990, firmado en 1987), Elias intenta relativizar la cuestión metodológica individualismo/holismo invitándonos a pensar el tema dinámicamente: las relaciones entre el individuo y su sociedad no siempre han sido iguales, y hay momentos históricos muy diversos en los que los dos polos del enunciado (Yo/Nosotros) se equilibran o desequilibran y hacen así más o menos relevantes los análisis más individualistas o más holistas. Nos dice, por ejemplo: "El equilibrio entre la identidad del yo y la identidad del nosotros ha experimentado un cambio notable desde la Edad Media europea; cambio que, muy brevemente, podría resumirse así: antes el equilibrio entre la identidad del nosotros y la identidad del yo se inclinaba más hacia la primera. A partir del Renacimiento el equilibrio empezó a inclinarse cada vez más hacia la identidad del yo» (N. Elias, 1990, p. 226).

Esto podría ser una forma de eludir la cuestión (por otra parte, perfectamente eludible, por cuanto que el modo en que se plantea en los artículos específicos de metodología de la ciencia no tiene nada que ver con la resolución de problemas reales de investigación, y sólo añade confusión a un tema que debe resolverse en el nivel de las interpretaciones de fenómenos, de planteamientos de investigación, de modelos analíticos, etc., es decir, en los procesos reales de investigación), pero en Elias es un planteamiento que quiere ser serio: la relatividad de la relación del Individuo con su Sociedad relativiza, a su vez, la cuestión metodológica. Esta es su tesis central sobre el problema y el eje del artículo citado. Podría tener mucho que ver esta dinamización del tema metodológico y su encuadre histórico con las tesis de Albert O. Hirschmann sobre la existencia de ciclos de dominio del interés público sobre el privado, y 
viceversa (A. Hirschmann, 1986, p. 11): «... nos llevan a preguntarnos si nuestras sociedades estarán predispuestas de algún modo hacia las oscilaciones entre períodos de intensa preocupación con los problemas públicos y de casi total concentración en las metas del mejoramiento individual y el bienestar privado». Tales planteamientos relativizarían la misma consistencia (su extensión y su intención) del concepto "sociedad», y lo harían, en su versatilidad empírica, más aceptable que un concepto tan metafísico como el que evoca su uso habitual en la tribu de los científicos sociales. En este sentido, la reivindicación —que a veces parece excesiva, como veremos- del concepto «sociedad» por parte de Elias se hace también desde un planteamiento revisionista del mismo, lo que otorga un interés especial a sus tesis.

\section{CONTRA MAX WEBER}

No es extraño que Elias dirija sus críticas contra Max Weber, aunque justifique sus puntos de vista en el momento histórico en que Weber trabaja: «Esto - se refiere Elias a la humanidad supraestatal como nueva unidad social determinante- se corresponde con el hecho de que hoy en día el problema de la relación entre individuo y sociedad sea planteado, en algunos aspectos, de manera distinta a como lo era cincuenta años atrás. En aquel entonces la población era menos de la mitad de la que existe ahora; más exactamente: la población mundial era más o menos el 40 por 100 de la actual. El número de personas que viven actualmente no carece de importancia para el estudio teórico y práctico de la relación real entre individuo y sociedad. Hay 5.000 millones de individuos en el mundo. La sociedad humana, la humanidad, no es más que el conjunto de estos individuos. Pero estos 5.000 millones de individuos no deambulan por el mundo solos, ni en grupos aislados, como afirmaban algunas antiguas teorías sociológicas, entre ellas la teoría de la acción de Max Weber. Prácticamente todos estos seres humanos están organizados en agrupaciones más o menos estables» (ob. cit., p. 189). Dejando a un lado ahora la justeza o no de estas palabras sobre Weber, Elias parece imputar a los incrementos de población consecuencias en la cuestión central de la existencia o no de una "sociedad» y de los cambios en la relación entre el yo y el nosotros. Las tesis weberianas podrían haber sido ciertas en su momento, aunque no ahora, y tales tesis describirían, siguiendo la lógica de Elias, un estadio preactual en el que el individuo era así, atómico e individualista. Lo cierto es que Weber ya tenía ante sí un mundo "moderno", con todas las consecuencias, y el sentido de sus tesis metodológicas no parece relativizable sólo al momento en que las escribe. Pero Weber le sirve didácticamente para subrayar su énfasis en la sociedad como concepto contextual-explicativo de fenómenos empíricos, y empírico-biológicos, como el lenguaje. En su The Symbol Theory (1991, pp. 20-21), y continuando con su tarea crítica bajo la forma, frecuentemente, de una sociología de la sociología, intentando explicar sociológicamente las posiciones 
metodológicas de los sociólogos, dice: «... a fact which is of some importance for the understanding of the symbol theory, introduced in the followings pages. This is the fact that, in reality, biological and social processes depend on each other» (la falta de conexión entre ambos procesos la atribuye Elias a la compartimentada especialización académica de sociólogos y biólogos), y sigue, centrándose en el individualismo metodológico como reflejo de una época: «... in this case is not the present type of academic specialization which causes the trouble, but the prototypical way in which people experience themselves in the more developed societies of our age and the way this self-image finds expression in the theories of social sciences. The self-image to which I refer is that of oneself and, generally, of all human beings as essentially independents actors. Twentieth-century sociology offers some well-know examples of this self-image. Weber's and Parsons's action theories are among the best known of these examples. In the form of a theory of communicative action they have been extended by Habermas with special regard to the problem of human communication. But it would be a mistake to attribute the blockage of knowledge which one can observe in all these theories, only or mainly to the authors of these theories. They are spokepersons and representative of a distinctive social habitus which is characteristic of our age. It induces in people the feeling that in some sense their individual self and, by proxy, every other individual, exist as a kind of monad independently of all others in a central position in the world and that one can explain all social events, including human communication, in terms of individual action» (cursivas mías). Quizá se pueda imputar a Elias parcializar su objetivo, pues una parte de la sociología contemporánea se escora más hacia posiciones holistas o hiperholistas que propiamente individualistas, y lo que se refleja en ese holismo no es tanto el supuesto individualismo de nuestro mundo como los importantes residuos comunitarios y aun autoritarios de la sociedad feudal: puestos a hacer metasociología, es probable que cierta forma de sociología sin sujeto no sea otra cosa que la permanencia de esos residuos en el discurso científico.

\section{SOCIEDAD, ESTADO Y METODO}

En un trabajo reciente (F. Bouza, 1993) he querido subrayar el carácter complejo del término "sociedad», sobre el que nace la sociología, en particular la sociología comtiana: no es un concepto inocente; ningún concepto lo es, y mucho menos en el marco del debate metodológico. Me interesa traer ahora y aquí mi reflexión de entonces porque en algunos aspectos parece no coincidir con la de Elias y, sin embargo, son perspectivas temporalmente complementarias: la reflexión de Elias (ob. cit., pp. 236, 237, 238) sobre la Sociedad y el Estado se hace desde la contraposición histórica entre el viejo régimen (el Estado: "El Estado soy yo») y la burguesía emergente (la Sociedad, o la Sociedad Civil); en estas condiciones, el concepto "sociedad» adquiere especia- 
les connotaciones antiestatales: "Hablantes de las clases medias en ascenso empleaban términos como "sociedad civil" y finalmente "sociedad" en general como medio de combate intelectual en sus confrontaciones con las clases altas de su época, con los príncipes y nobles, quienes monopolizaban los medios de poder estatales. La connotación de una diferencia, quizás hasta de una oposición entre Estado y sociedad, que hace parecer que el Estado es algo exterior a la sociedad, y la sociedad algo exterior al Estado, todavía no ha desaparecido por completo del uso de esos términos» (ob. cit., p. 237). Pero, para completar el proceso, habría que añadir que cuando la sociología se inicia con ese nombre, la tarea de Comte es, precisamente, la fusión sociedad/Estado a través de una metodología que unifica el concepto «sociedad» con el Estado emergente y funda una visión holística, sobre bases políticas, del objeto sociológico, al margen del arranque empírico (el individuo), coincidiendo con la emergencia del Estado postrevolucionario y con la influencia sobre ese Estado del pensamiento conservador en su lucha contra el individualismo liberal, en liza ambas formas de entender el nuevo mundo por el control del proceso histórico en curso. $\mathrm{Si}$ subrayo esto es porque me parece insuficiente el planteamiento de Elias sobre el concepto «sociedad", mostrando sólo una de sus caras. La síntesis de mis puntos de vista, que ahora quiero confrontar, sería ésta: «Hay un aspecto político en ese esencialismo positivista que busca en "la sociedad", bajo supuesta bandera sociometodológica, la entronización del Estado nuevo, que se anuncia poderoso, como Weber teorizará algo más adelante: la sociedad no es tanto un tópico de método como una simbolización cientista del estado burocrático. Los conservadores como Burke, De Maistre, De Bonald o Lamennais, atacaron al individuo de la nueva época y contraatacaron al nuevo orden mitificando a "la sociedad" como una exigencia de orden superior al individuo. El mismo Comte explicita en su obra la influencia del pensamiento conservador, en particular de De Maistre y de De Bonald. La polémica del individualismo metodológico nacerá junto a la polémica del individualismo político: no son la misma cosa, pero parecen ir juntos» (F. Bouza, ob. cit., p. 198).

Estos dos momentos (primero, la Sociedad contra el Estado: la «sociedad civil»; y segundo, la Sociedad es el Estado: el Estado fuerte, burocrático, o, más tarde, el Estado del Bienestar, como forma providencial y paternal de fortaleza) definen marcos diversos de la relación yo/nosotros y/o de la relación individuo/sociedad, al tiempo que nos permiten situar el nacimiento del holismo sociológico en ese punto histórico en el que el pensamiento conservador propone un modelo antiindividualista al estado nuevo. Y aquí retomo los puntos de vista de Elias.

\section{CAMBIOS EN EL EQUILIBRIO ENTRE EL YO Y EL NOSOTROS}

Traspasando sus preocupaciones metodológicas a un plano ético, Elias unifica sus tensiones sociológicas en un objetivo humanista que resume su trabajo: 
«La pregunta es si el desarrollo de la humanidad, de la forma global de la convivencia humana, ha alcanzado ya o puede alcanzar un nivel en el cual sea posible conseguir que predomine entre los seres humanos un perfecto equilibrio entre el yo y el nosotros» (ob. cit., p. 232; cursivas mías). Elias ha transformado su objetivo sociológico en objetivo ético universal: lo ha convertido en imperativo categórico. En cierto modo, la impresión que produce esto es la de que la cuestión metodológica no es en Elias otra cosa que una metáfora del que realmente es el sustrato moral de su trabajo científico: la consecución de una sociedad equilibrada entre lo individual y lo colectivo. Ciertamente, como síntesis de todos los desiderata de la ciencia política y moral, la utopía armónica de Elias es perfecta. Podría ser asumida, a ese nivel de abstracción, por John Rawls (1990, pp. 43-44): «La conexión entre la idea de cooperación social y la concepción de la persona que voy a presentar puede expresarse del siguiente modo. La idea de cooperación social no es simplemente la de la actividad social coordinada y eficientemente organizada y guiada por reglas públicamente reconocidas para alcanzar algún fin general. La cooperación social es siempre en beneficio mutuo y esto implica que la cooperación supone dos elementos: el primero es una noción compartida de términos equitativos de cooperación, que puede esperarse razonablemente acepte cada participante, siempre que alguien más los acepte igualmente. Los términos equitativos de cooperación articulan una idea de reciprocidad y acción mutua: todos los que cooperan deben beneficiarse, o compartir las cargas comunes... La unidad de cooperación social se basa en personas que concuerdan en su noción de términos equitativos» (cursivas mías). Aunque las tesis de Rawls aparecen algo más escoradas hacia el «nosotros». Más dudoso es que Elias coincidiera con otro clásico contemporáneo, Richard Rorty (R. Rorty, 1991, p. 17), cuya fractura entre «sí mismo" y "solidaridad humana» evoca el mismo reproche que Elias hace a Parsons, con su supuesta desconexión entre «ego» y «sistema»: "Este libro — dice Rorty - intenta mostrar el aspecto que cobran las cosas si excluimos la exigencia de una teoría que unifique lo público y lo privado y nos contentamos con tratar las exigencias de creación de sí mismo y de solidaridad humana como igualmente válidas, aunque definitivamente inconmensurables.» $\mathrm{O}$ bien (ob. cit., p. 216): «En resumen, deseo distinguir entre la solidaridad humana como identificación con "la humanidad como tal" y la solidaridad como la duda respecto de sí mismo... La identificación me parece imposible: una invención de filósofos, un torpe intento de secularizar la idea de llegar a ser uno como Dios» (cursivas mías). La suave transición entre el yo y el nosotros aparece aquí impugnada, al menos a la manera en que Elias la plantea.

La familia y el lugar de nacimiento son los focos primarios más antiguos del «nosotros» (N. Elias, 1990, p. 205). A partir de esta premisa, Elias compara el desarrollo social con los cambios en el equilibrio entre el yo y el nosotros: «Si se considera la relación entre la identidad como yo y la identidad como nosotros, bien podría decirse que en todos los países, tanto en los más como en los menos desarrollados, existen ambos tipos de identidad, pero que en los pri- 
meros la intensidad de la identidad como yo es comparativamente mayor y, por consiguiente, en los paises menos desarrollados es más intensa la identidad preestatal como nosotros, lo mismo si ésta remite a la familia, al lugar de nacimiento o incluso a la tribu» (ob. cit., p. 205; cursivas mías).

Ahora bien, en los procesos de expansión de las unidades estatales, ya sea al estado nacional clásico, ya a las unidades continentales o a la misma humanidad, los procesos de pérdida del nosotros previo para adoptar el nuevo nosotros van acompañados de diversos fenómenos de desarraigo e individualización: "... en un determinado nivel del proceso de formación de un estado se fomenta la individualización, la mayor acentuación de la identidad como yo del ser humano particular y el apartamiento de éste de las agrupaciones tradicionales... En la hasta ahora última etapa del desarrollo, el proceso de formación de Estados conduce hacia una individualización de las masas" (N. Elias, ob. cit., pp. 206, 207, 208; cursivas mías).

La formación de unidades superiores de identidad exigiría la adopción de un nuevo "nosotros», y eso es complicado porque "... en comparación con el cambio relativamente rápido producido por el movimiento integrador, el correspondiente cambio de la actitud social de las personas implicadas es extraordinariamente lento. Estructuras sociales de la personalidad de los seres humanos singulares, en particular la concepción del yo y del nosotros, son relativamente duraderas y tenaces. Se resisten a las múltiples novedades que trae consigo la transición a un nuevo nivel de integración» (ob. cit., pp. 247248).

Los conceptos «actitud social» y «estructura social de la personalidad» son los conceptos-puente, para Elias, entre el yo y el nosotros, entre la sociedad y el individuo, y ellos explican cosas como el idioma o el carácter nacional (y es curioso cómo Elias asume este último concepto, tan cuestionado, al menos enunciado como «carácter»): «... se advierte que los rasgos de la identidad grupal nacional, es decir, lo que llamamos "carácter nacional", están profunda y firmemente integrados en la estructura de la personalidad de las personas particulares como una capa más de la actitud social» (ob. cit., p. 242). Para explicar la persistencia de las mentalidades más allá de los cambios de nivel de integración, Elias recurre a conceptos individualistas, si bien marcados con el adjetivo «social» para subrayar su dependencia de los procesos grupales. A pesar de estos volatines metodológicos que realiza el sociólogo para salvar la conexión individuo/sociedad, él mismo acepta, de hecho, el abismo que puede separar las nuevas estructuras sociales de lo que él llama "actitud social» — "cada persona lleva en sí la actitud de un grupo» (ob. cit., p. 210) — o respuesta del yo a los cambios, por más que en esa respuesta haya, cómo no, elementos sociales. $\mathrm{Y}$ aquí reaparece el Elias que quiere salvar para la sociología el nivel analítico del yo: «El concepto de actitud social no forma parte aún del conjunto de conocimientos teóricos elementales que los profesores de sociología y de las otras ciencias sociales presentan a las generaciones más jóvenes para orientarlas en lo referente a la sociedad humana. La profunda raigambre de las diversas 
características nacionales y, muy ligada a esto, la conciencia de la propia identidad del nosotros en cuanto nación, son ejemplos que ilustran hasta qué punto puede la actitud social del individuo servir de base para el desenvolvimiento de diferencias absolutamente personales e individuales. La individualidad del inglés, el holandés, el sueco o el alemán constituye un cierto modo de elaboración personal de una actitud social común, o, en este caso, de una actitud nacional» (ob. cit., p. 243).

\section{EXPLICACIONES DESDE EL MODELO DE ELIAS: NACIONALISMO, XENOFOBIA, MODERNIZACION, JAPONIZACION, CORRUPCION}

La pareja conceptual yo/nosotros y sus equivalentes en diversos niveles analíticos, tal como la maneja Norbert Elias, permite explicar sencilla y brillantemente algunos acontecimientos tan interesantes como los que se enuncian en este parágrafo. Hay que tener gran cautela, sin embargo, si se desciende a niveles empíricos inmediatamente contrastables: los enunciados de Elias están elaborados desde una perspectiva macrosociológica y son muy sugerentes para esos grandes procesos, pero no son inmediatamente traducibles a los cambios cotidianos (y aquí pueden servir de ayuda los interesantes planteamientos metodológicos de Charles Tilly sobre las "grandes estructuras, procesos amplios, comparaciones enormes» - Ch. Tilly, 1991-), advertencia ésta tanto más necesaria cuanto que buena parte de la sociología tradicional se ha movido como pez en el agua a través del análisis "macro», con sonoros despistes analíticos en el mundo terrestre o concreto, al igual que la economía.

Dicho esto, parece cierto que el modelo de análisis de partida que nos proporciona Elias permite explicaciones sencillas y relativamente contrastables. El nacionalismo no es otra cosa que la adhesión a un nosotros, adhesión que nosotros podríamos dividir, en una primera aproximación, en normal y patológica. De una adhesión anormal o patológica derivaría la xenofobia, el horror a lo diferente, el racismo, etc. Admitiendo, para completar el modelo, que una adhesión de este tipo debe tener bases no sólo en la cultura, sino en los procesos más materiales, como los económicos. Una adhesión de este tipo se caracterizaría por la renuncia o rechazo a más altos niveles de integración. Demasiado sencilla y amplia esta explicación-corolario desde las tesis de Elias para ser cierta, pero también clara y manejable para proponer elementos de contrastación. No se puede renunciar a ella.

Es sabido que los procesos de modernización ocurren en mejores condiciones si se da una conciencia nacional fuerte, y que un país con problemas de integración interior irresueltos sufre retrasos y problemas en mucha mayor medida que un país bien integrado: un «nosotros» claro y asumido es un factor de buena salud modernizadora. Así, paradójicamente, y uniéndolo a otras tesis contrastadas, la unión de un yo fuerte y respetado desde el estado (un indivi- 
duo reconocido como tal) y de un nosotros fortalecido desde el legendario colectivo ayudarían extraordinariamente a mantener los procesos de modernización y desarrollo. También esto es colegible como corolario de Elias y relativamente cierto si buscamos ejemplos en el mundo contemporáneo, aunque con excepciones y matices. El mismo Elias aduce el curioso modelo japonés para establecer tales matices: «En el desarrollo del Japón, y quizá también de otros países asiáticos en vías de modernización, se plantea una cuestión muy particular. Allí el cambio del equilibrio entre el yo y el nosotros en favor de la identidad como yo es menos intenso que en los países occidentales, lo que otorga considerables ventajas a su capacidad para competir» (cursivas mías). La persistencia de un nosotros premoderno, unido a la presencia de un yo difuso pero real y otros factores diversos, parece propiciar ventajas competitivas. Quizá nos complete la explicación Ruth Benedict (1974, pp. 176-177) en sus conocidos análisis de los patrones de la cultura japonesa: «El modo japonés de enfocar la vida es justamente aquel que expresan las fórmulas del chu, ko, giri, jin y los sentimientos humanos. Para ellos, "el deber total del hombre" está parcelado, como un mapa que se divide en distintas provincias. Según afirman, la vida individual consiste en "el círculo de $c h u$ ", el "círculo de ko", el "círculo de giri", el "círculo de jin", el "círculo de los sentimientos humanos" y muchos más. Cada círculo tiene su código especial detallado... Los códigos, incluso dentro de cada círculo, están estructurados de tal manera que cuando las condiciones cambian puede ser necesario, y legítimo, un comportamiento muy distinto. El giri al señor de uno exigía lealtad suprema; pero si ofendía al servidor, cualquier traición era poca. Hasta agosto de 1945, el chu exigía que el pueblo japonés combatiera con el enemigo hasta caer el último hombre. Cuando el emperador cambió las exigencias del "chu" al proclamar por radio la capitulación del Japón, los japoneses se deshacian tratando de expresar su cooperación con los visitantes. Esto asombró a los occidentales. Según nuestra experiencia, la gente actúa "de acuerdo con su carácter"... Esperamos que tengan una ideología política y que, por tanto, luchen contra la ideología opuesta.»

Un «nosotros» de esta magnitud tiene la ventaja de la enorme cohesión que supone, pero tiende a inhibir la iniciativa individual: si no es así, y se compatibiliza (?) tal iniciativa con un grupo tal de cohesión, el rendimiento nacional, la capacidad productiva, los costes laborales y todas las magnitudes que pueden ser controladas desde un "nosotros» así, tenderán a incrementar la competitividad. Podría ser el caso del Japón, al menos en algún momento de su expansión económica. Tal unión de cultura y economía propiciaría ciertas ventajas, al contrario de lo que ocurre en USA, si creemos las tesis de Daniel Bell (D. Bell, 1982, pp. 11-12): «Las contradicciones que veo en el capitalismo contemporáneo derivan del aflojamiento de los hilos que antaño mantenían unidas la cultura y la economía, y de la influencia del hedonismo, que se ha convertido en el valor predominante de nuestra sociedad... La argumentación desarrollada en este libro es que los tres ámbitos - la economía, el orden político y la cultura- se rigen por principios axiales contrarios: la economía, por la eficiencia; el 
orden político, por la igualdad; y la cultura, por la autorrealización (o la autogratificación). Las disyunciones resultantes han moldeado las tensiones y los conflictos sociales de la sociedad occidental en los últimos 150 años.»

$\mathrm{Y}$ aún hay otro fenómeno de interés y actualidad, como el de la corrupción, al que Elias aplica sus funcionales esquemas de cambio social y de tensión yo/nosotros. La corrupción aparece como consecuencia de esos cambios y esas tensiones, al menos algún tipo de corrupción, aunque la ampliación del esquema de Elias para que abarque al fenómeno en toda su complejidad es una interesante tentación que no parece inviable: «El cambio de la identidad como nosotros que se verifica durante la transición de un nivel de desarrollo a otro también puede ser ilustrado como un conflicto de lealtades. La tradicional formación de la conciencia, el tradicional ethos de la adhesión a la unidad de supervivencia tradicional que es la familia, el clan —en suma: el grupo de parentesco, más reducido o más amplio-, exigen que un miembro del grupo más rico o mejor situado aporte alguna ayuda a sus parientes, aunque sean lejanos, cuando éstos la soliciten. Así, para los altos funcionarios de un nuevo Estado independiente es extremadamente difícil no apoyar a sus parientes si éstos intentan hacerse con un cargo público codiciado. Visto desde el ethos y la formación de la conciencia propios de los Estados más desarrollados, el favoritismo de que hace gala un funcionario elevado cuando concede un cargo público a algún pariente es una forma de corrupción. Desde la formación de la conciencia preestatal, esto es un deber y, en tanto que todos hacen lo mismo, es decir, en tanto que forma parte habitual de las luchas por el poder y el status de los clanes, es también una necesidad. Así, pues, en la transición hacia un nuevo nivel de integración se producen conflictos de lealtad y de conciencia que son al mismo tiempo conflictos de identidad... Con bastante frecuencia suele considerarse un mandato de la razón eterna el que en los países más desarrollados el otorgamiento de cargos públicos a parientes ceda el paso al otorgamiento de cargos públicos fundamentado en las aptitudes individuales de los solicitantes. Pero esto, que es realista, posible, necesario y, en este sentido, racional, puede no ser tal en otras etapas del proceso de desarrollo social» (N. Elias, 1990, pp. 206-207). Es un tema clásico de Max Weber, al que Elias da un fundamento mucho más individualista que el propio Weber, a pesar de las quejas de Elias que antes veíamos sobre la metodología de la acción social de Weber. En todo caso, ambas tesis se complementan y completan un cierto enfoque socioantropológico del tema de la administración premoderna o, en términos de Weber, de la dominación tradicional. En esta dominación, que es la que está presente en la anterior cita de Elias (que éste llamará tribal, familiar, preestatal, pero que la menciona en el interior del estado moderno emergente en los países en desarrollo), Weber (1979, p. 181) considera que «el cuadro administrativo típico puede ser reclutado de modo: a) tradicional, por lazos de "piedad" de los vinculados al señor ("reclutamiento patrimonial"): pertenecientes al linaje, esclavos, funcionarios domésticos, en particular: "ministeriales", clientes, colonos, libertos...". El nosotros preestatal de Elias está asentado sobre la red de vínculos diversos que 
Weber explicita en su trabajo clásico (una auténtica sociología de los procesos, como quería Elias).

\section{PARA ENTENDER A ELIAS}

Este interesantísimo trabajo de Elias sobre las relaciones entre el yo y nosotros expresa, a modo de coda de toda su obra, la sustancia primigenia de su preocupación metodológica, cuyo último deseo no es otro que el de resituar a la sociología en el entendimiento de los procesos y acercarla a la historia material y a la historia de la cultura desde una perspectiva de acatamiento de la autonomía de lo social como campo analítico y método propio, al tiempo que no se renuncia al uso de variables individuales o mentales ni a su interacción con variables propiamente sociales. Estos deseos y estos métodos conforman, además, toda una versión ética del debate metodológico: aquella ética que Elias fundamenta en el «equilibrio entre el yo y el nosotros».

\section{BIBLIOGRAFIA}

BELL, D. (1982): Las contradicciones culturales del capitalismo, Alianza, Madrid.

Benedict, R. (1974): El crisantemo y la espada. Patrones de la cultura japonesa, Alianza, Madrid. BouzA, F. (1992): "Orden social y orden sociológico», en Escritos de Teoría Sociológica en Homenaje a Luis Rodríguez Zúñiga, pp. 179-201, CIS, Madrid.

Elias, N. (1990): "Cambios en el equilibrio entre el yo y el nosotros», en La sociedad de los individuos, Península, Barcelona.

- (1991): The Symbol Theory, SAGE, Londres.

Hirschmann, A. O. (1986): Interés privado y acción pública, FCE, México.

RAWLS, J. (1990): Sobre las libertades, Paidós, Barcelona.

RORTY, R. (1991): Contingencia, ironía y solidaridad, Paidós, Barcelona.

Tilly, Ch. (1991): Grandes estructuras, procesos amplios, comparaciones enormes, Alianza, Madrid. Weber, M. (1979): Economía y Sociedad, FCE, México. 


\title{
RESUMEN
}

El artículo de Elias sobre las relaciones entre el yo y nosotros expresa, a modo de coda de toda su obra, la sustancia primigenia de su preocupación metodológica, cuyo último deseo no es otro que el de resituar a la sociología en el entendimiento de los procesos y acercarla a la historia material y a la historia de la cultura desde una perspectiva de acatamiento de la autonomía de lo social como campo analítico y método propio, al tiempo que no se renuncia al uso de variables individuales o mentales ni a su interacción con variables propiamente sociales. Estos deseos y estos métodos conforman, además, toda una versión ética del debate metodológico: aquella ética que Elias fundamenta en el «equilibrio entre el yo y el nosotros».

\begin{abstract}
The article of Elias about the relationship between the «I» and «we» is the expression of the very core substance of his methodological interest, a recapitulation of his whole work. His final purpose is to relocate sociology within the understanding of processes and to bring it near to material and cultural history. Accepting the autonomy of the social as an analytical field with a distinctive methodology, Elias does not renounces to the use of individual or mental variables, nor their interaction of both with social variables. These interests and methods shape an ethical point of view of the methodological debate: the ethics that Elias establishes within "the equilibrium between the I and we».
\end{abstract}


NOTAS DE INVESTIGACION 